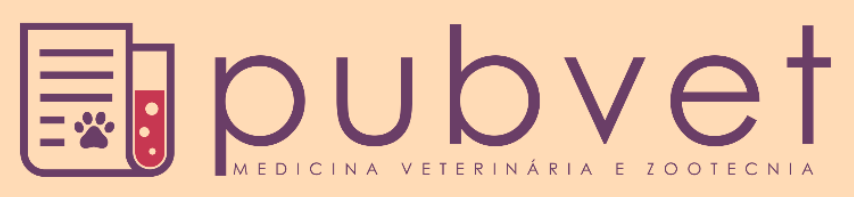

https://doi.org/10.31533/pubvet.v13n01a248.1-6

\title{
Diagnóstico de Dioctophyma renale em um cão na baixada santista através da ultrassonografia abdominal
}

\author{
Caroline Corrêa De Tullio Augusto Roque ${ }^{1 *}$, Catharina da Rocha Brito ${ }^{2}$, Magda \\ Regina $^{3}$, Paloma Paim Taboada ${ }^{4}$, Andréa Regina Abrantes Gomes ${ }^{30}$, Mariella Baldini ${ }^{50}$, \\ Luciana Casartelli Alves $^{6}{ }^{\bullet}$, Larissa de Oliveira Taboada ${ }^{4}$
}

${ }^{1}$ Médica Veterinária, MSc, proprietária e funcionária da empresa Ômega Imagem Veterinária, Santos-Sp, Brasil

${ }^{2}$ Médica Veterinária autônoma, MSc, Santos-Sp, Brasil.

${ }^{3}$ Médica Veterinária da Coordenadoria de Defesa da Vida Animal, MSc, Santos-Sp, Brasil.

${ }^{4}$ Médica Veterinária da Coordenadoria de Defesa da Vida Animal, Santos-Sp, Brasil.

${ }^{5}$ Médica Veterinária autônoma, Santos-Sp, Brasil

${ }^{6}$ Médica Veterinária, PhD, da Coordenadoria de Defesa da Vida Animal, Santos-Sp, Brasil.

*Autor para correspondência, E-mail: carolimagemvet@gmail.com

Resumo. O Dioctophyma renale (D. renale) é um helminto que habita preferencialmente o rim direito do hospedeiro definitivo, mas descrito também em tecidos peri-renais, ureteres, bexiga, uretra, bolsa escrotal, tecido subcutâneo inguinal, útero, ovários, linfonodos mesentéricos, glândula mamária, cavidade torácica, pericárdio, cavidade abdominal, estômago e fígado. Por apresentar comportamento destrutivo progressivo, pode levar à perda total do órgão afetado. Os sintomas são por muitas vezes, inespecíficos, variando de acordo com a região afetada, e muitas vezes podem ser inexistentes. Sua incidência está relacionada ao acesso a ambientes aquáticos e hábitos alimentares do animal, como por exemplo, ingestão de peixe ou rã cruas. Objetivou-se com esse trabalho, relatar um caso de Dioctophyma renale em um cão habitado na cidade de Santos, área não endêmica do parasito, a fim de alertar a população, incluindo os médicos veterinários, da possibilidade de investigação e riscos desta doença. O paciente relatado é uma cadela, sem raça definida, de aproximadamente 3 anos, que começou a apresentar prostração e hematúria progressiva observados em consulta clínica. Foi solicitado hemograma e ultrassonografia abdominal, sendo este último exame o qual identificou o nematoide no interior do rim direito. A nefrectomia foi realizada no dia seguinte ao diagnóstico e atualmente o paciente passa bem.

Palavras chave: Dioctophyma renale, helminto, rim direito, ultrassom

\section{Diagnosis of Dioctophyma renale in a dog in the Santos basin by abdominal ultrasonography}

\footnotetext{
Abstract. Dioctophyma renale is a helminth that preferably inhabits the definitive host's right kidney, but is also described in peri-renal tissues, ureters, bladder, urethra, scrotum, inguinal subcutaneous tissue, uterus, ovaries, mesenteric lymph nodes, mammary gland, thoracic cavity, pericardium, abdominal cavity, stomach and liver. By presenting progressive destructive behavior, it can lead to total loss of the affected organ. The symptoms are often unspecific, varying according to the affected region, and may often be non-existent. Its incidence is related to access to aquatic environments and feeding habits of the animal, such as fish or raw frog ingestion. The objective of this study was to report a case of Dioctophyma renale in a dog inhabited in the city of Santos, a non-endemic area of the parasite, in order to alert the population, including veterinarians, of the possibility of research and risks of this disease. The reported patient is a female dog, with no defined breed of approximately 3 years old, which began to present prostration and progressive hematuria observed in
} 
clinical consultation. Hemogram and abdominal ultrasonography exams were requested; however the latter one was the exam that identified the nematoid inside the right kidney. The nephrectomy was performed the day after the diagnosis and the patient is currently well.

Key words: Dioctophyma renale, helminth, right kidney, ultrasound

\title{
Diagnóstico de Dioctophyma renale en un perro en la bajada santista a través de la ultrasonografía abdominal
}

\begin{abstract}
Resumen. El Dioctophyma renale es un helminto que habita preferentemente el riñón derecho del huésped definitivo, pero también habita los tejidos peri-renales, uréteres, vejiga, uretra, bolsa escrotal, tejido subcutáneo inguinal, útero, ovarios, ganglios linfáticos mesentéricos, glándula mamaria, cavidad torácica, pericardio, cavidad abdominal, estómago e hígado. Por presentar comportamiento destructivo progresivo, puede llevar a la pérdida total del órgano afectado. Los síntomas, son a menudo inespecíficos, variando de acuerdo con la región afectada, y muchas veces pueden ser inexistentes. Su incidencia está relacionada con el acceso a los ambientes acuáticos y los hábitos alimenticios del animal, como, por ejemplo, la ingestión de peces o ranas crudos. El objetivo de este trabajo fue relatar un caso clínico de Dioctophyma renale en un perro proveniente de la ciudad de Santos, área no endémica del parásito, a fin de alertar a la población, incluidos los médicos veterinarios, de la posibilidad de investigación y riesgos de esta enfermedad. La paciente relatada era una perra criolla con edad de aproximadamente 3 años, que comenzó a presentar postración y hematuria progresiva observados en consulta clínica. Se solicitó hemograma y ultrasonografía abdominal, siendo este último examen el cual identificó el nematodo en el interior del riñón derecho. La nefrectomía fue realizada al día siguiente del diagnóstico y actualmente la paciente pasa bien.
\end{abstract}

Palabras clave: Dioctophyma renale, helminto, riñón derecho, ultrasonido

\section{Introdução}

Primeiramente descrito por Goeze, 1782 (Beaver \& Theis, 1979), o Dioctophyma renale é um helminto que habita tecido renal preferencialmente o direito. Este parasita também é descrito em tecidos peri-renais, ureteres, bexiga, uretra, bolsa escrotal, subcutâneo inguinal, útero, ovários, linfonodos mesentéricos, glândula mamária, cavidade torácica, pericárdio, cavidade abdominal, estômago e fígado (Barriga, 2002; Dacorso Filho et al., 1954; Leite et al., 2005; Miranda et al., 1992; Nakagawa et al., 2007). Quando parasitado nos rins, apresenta caráter de destruição progressiva levando o órgão apresentar apenas cápsula fibrosa (Leite et al., 2005). Conhecido como verme renal gigante é o maior nematóide conhecido pertencente à ordem Enoplida, família Dioctophymatidae (Barriga, 2002) cujo comprimento e largura podem atingir até $100 \mathrm{~cm}$ e 0,4 a 1,2 cm, respectivamente. O macho apresenta estriações transversais finas na cutícula cujo comprimento médio é de 14 a $45 \mathrm{~cm}$, boca circundada por coroa de seis papilas, espículos em forma de cerda, com 5 a $6 \mathrm{~mm}$ de comprimento e bolsa copuladora robusta em forma de sino. As fêmeas, por sua vez, apresentam a cutícula estriada e 20 a $100 \mathrm{~cm}$ de comprimento. Ambos apresentam coloração avermelhada secundária à hematofagia.

O D. renale apresenta distribuição mundial, com parasitose descrita em carnívoros domésticos como cães (Canis lupus familiaris) (Kano et al., 2003; Kommers et al., 1999; Leite et al., 2005; Monteiro et al., 2002; Pereira et al., 2006) e gatos (Felis catus) (Verocai et al., 2009); acometendo também espécimes selvagens como: ferrets (Mustela putorius furo) (Barriga, 2002; Dyer, 1998; Mech \& Tracy, 2001; Zabott et al., 2012) (Crysocyon brachyurus), bicho preguiça (Choloepus didactylus), lontras (Lutra longicaudis), cachorro do mato (Cerdocyon thous) (Kommers et al., 1999), quati (Nasua nasua) (Milanelo et al., 2009), macaco-prego (Cebus apella) (Ishizaki et al., 2010) e tartaruga tigre d'água (Trachemys dorbigni) (Mascarenhas \& Müller, 2015). A incidência do parasito em animais está relacionada ao acesso a ambientes aquáticos (Kano et al., 2003), seus hábitos alimentares (Santos \& Beisiegel, 2006), e ao ciclo de vida do parasita (Kano et al., 2003). Este nematoide apresenta um ciclo biológico heteroxeno o qual envolve mais de um hospedeiro para finalizar seu desenvolvimento. 
Inicialmente, os ovos, elipsoides de casca espessa com pequenas depressões contendo o primeiro estágio larval (L1), são encontrados na urina dos animais parasitados. O desenvolvimento da fase L1 ocorre em aproximadamente quatro semanas quando são ingeridos por um anelídeo oligoqueta aquático (Lumbriculus variegatus) (Barriga, 2002; Kommers et al., 1999). Ao eclodirem no intestino dos hospedeiros intermediários, as larvas, após dois meses sofrem metamorfose em larvas infectantes (L2). Essas podem ser ingeridas por girinos, rãs ou peixes que servem como hospedeiros paratênicos ou de transporte até serem ingeridas pelos hospedeiros permanentes as quais podem permanecer vivas por até 5 anos nos rins (Vibe, 1985). Essa parasitose possui relevância pelo fato de acometer humanos por meio da ingestão de produtos intermediários, crus ou inadequadamente cozidos, ou hospedeiros paratênicos (anelídeos, peixes de água doce e/ou sapos) contendo larvas infectantes (Kommers et al., 1999; Measures \& Anderson, 1985). Nos que se refere à manifestação em animais suscetíveis, os sinais clínico se manifestam por apatia, abatimento, inapetência e emagrecimento, porém muitas vezes pode não apresentar nenhuma sintomatologia (Colpo et al., 2005). Os sintomas mais específicos variam conforme a localização do parasita. Ao parasitar rins, é possível observar arqueamento dorsal, hematúria, estrangúria e marcha alterada, muitas vezes confundida com outras patologias (Colpo et al., 2005; Dacorso Filho et al., 1954). O diagnóstico da presença do helminto pode ser feito pelo achado de ovos operculados de casca espessa e rugosa na urina, por radiografia ou ultrassonografia da cavidade abdominal (Barriga, 2002).

Neste contexto, objetivou-se relatar um caso de $D$. renale em um cão habitado na cidade de Santos, área não endêmica e de ocorrência ainda não descrita, a fim de alertar a população quanto aos riscos e prevenção da doença e também mostrar aos médicos veterinários a possibilidade de ocorrência na região.

\section{Relado de caso}

Um cão fêmea, sem raça definida, de aproximadamente 3 anos de idade e pesando em torno de 10 quilos foi atendida pela Coordenadoria de Defesa da Vida Animal (CODEVIDA) no município de Santos no dia 5 de julho de 2018. O animal foi adotado com aproximadamente 1 ano de vida em dezembro de 2016 no município Pedro de Toleto, localizado na região geográfica imediata de Santos e levado para esta cidade, aonde os novos tutores residem.

Após dois dias de apatia e hiporexia, a paciente começou a apresentar hematúria intensa e foi levada à CODEVIDA pelo seu tutor. Ao exame físico, constatou-se temperatura baixa no valor de $37,5^{\circ} \mathrm{C}$, abdominalgia e esplenomegalia. Foi realizado urinálise, hemograma, perfil renal e hepático. Observouse alteração no hemograma como trombocitopenia em um valor de 85 mil plaquetas (ref.: entre 200$500 \mathrm{mil} / \mathrm{mm}^{3}$ ) e leucocitúria, 300.000 (ref. 0-10.000MI). Dentre as suspeitas clínicas estavam babesiose, anaplasmose e erliquiose, porém pela hipotermia ser um sinal atípico nessas moléstias, foi solicitado exame ultrassonográfico abdominal para melhor investigação clínica. O exame ultrassonográfico foi realizado no Centro de Diagnóstico por Imagem, Ômega Imagem Veterinária, em Santos.

Através deste exame, foi constatado importante esplenomegalia, além de acentuado aumento de volume em rim direito, medindo em torno de $5,34 \mathrm{~cm}$ em eixo crânio caudal e $3,67 \mathrm{~cm}$ em eixo ventrodorsal, com contornos precisos e levemente irregulares, com perda maciça da sua arquitetura interna em virtude a presença de inúmeras estruturas tubulares, hipoecogênicas com parede hiperecogênica, calibre variando entre $0,48 \mathrm{~cm}$ e $0,51 \mathrm{~cm}$ entremeadas ao conteúdo ecogênico (Figura 1), as quais apresentavam discreta mobilidade e ausência de sinal em Doppler colorido, ocupando toda a extensão renal e contidas apenas pela cápsula renal espessada. Destacou-se também, aumento da ecogenicidade em mesentério adjacente, compatível com processo inflamatório em gordura mesentérica peri-renal. No tocante ao rim esquerdo, este apresentou dimensões e aspectos ultrassonográficos dentro dos limites da normalidade, assim como os demais órgãos abdominais avaliados.

Em razão desses achados, a paciente foi encaminhada para nefrectomia direita no dia seguinte. Macroscopicamente o rim estava hemorrágico apresentando moderado sangramento adjacente, dificultando a diferenciação imediata entre artéria e veia renal, além da presença de inúmeras pequenas áreas hemorrágicas (petequeias) em todo peritônio e em gordura mesentérica. Foi realizada lavagem abdominal com solução fisiológica morna. Em seguida, foi realizada incisão paracostal direita para acesso cirúrgico no rim direito, conforme técnica descrita por Fossum (2014). Após remoção do órgão foi realizada a abertura e dois grandes helmintos foram visualizados. Não havia parênquima renal viável, 
apenas cápsula renal difusamente espessada e irregular em pelve. Após o tratamento cirúrgico a paciente se encontra clinicamente e laboratorialmente bem.

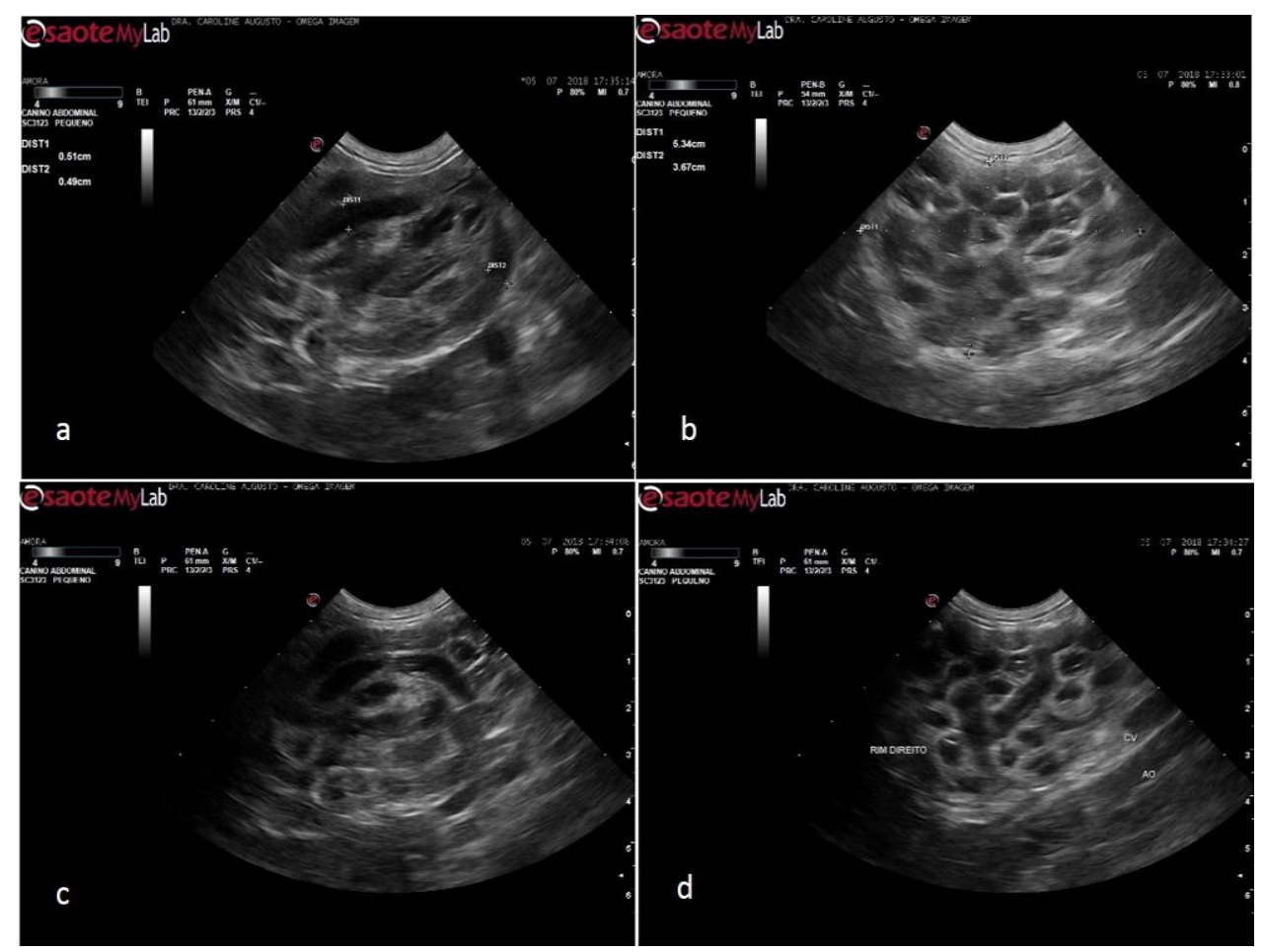

Figura 1. (a) Imagem ultrassonográfica em corte longitudinal de dois helmintos, com espessura de $0,51 \mathrm{~cm}$ e $0,49 \mathrm{~cm}$; (b) acentuado aumento de volume em rim direito medindo $5,34 \mathrm{~cm}$ em eixo crâniocaudal e 3,67cm em eixo ventro-dorsal; (c-d) imagens ultrassonográficas demonstram perda maciça da arquitetura interna renal em virtude da presença de inúmeras estruturas tubulares, hipoecogênicas com parede hiperecogênica entremeadas.

\section{Discussão}

No presente trabalho, o exame ultrassonográfico em região abdominal se mostrou bastante eficiente na detecção precoce do parasita, sendo imprescindíveis no diagnóstico definitivo e direcionamento para o tratamento adequado, que inclui a ressecção total do rim acometido, contribuindo para um bom prognóstico, assim como relatado por Cottar et al. (2012) e Zardo et al. (2012).

As alterações que esse parasita provoca são bem características como presença de inflamação, incluindo a destruição progressiva do parênquima renal, tornando o rim uma capsula fibrosa (Ferreira et.al, 2010). O nematoide destruiu totalmente o parênquima renal do paciente canino e a cápsula renal estava difusamente espessada e irregular em região da pelve, como descrito por Leite et al. (2005). Há relatos de identificação de ovos presentes em urina de cães infectados contaminando coleções d'agua em centros urbanos (Colpo et al., 2005; Mascarenhas \& Müller, 2015; Milanelo et al., 2009), o que não foi verificado neste caso. Além disso, já foi relatado que cães errantes são caçadores não seletivos e ratos podem atuar como hospedeiros paratênicos, servindo de fonte de infecção para esses (Kommers et al., 1999).

No exame de sedimento urinário e ascitico podem demonstrar ovos de D. renale (Neves, 2005); porém não foram visualizados no exame parasitológico de urina do presente paciente, possivelmente devido à ausência de parasitos fêmea grávida, presença de foras imaturos, tempo de exposição e ao próprio organismo imunidade e resistência do animal.

A paciente ainda demonstrou a predominância de parasitas no rim direito assim como relatado por diversos autores ao longo dos anos, como Dacorso Filho et al. (1954), Giovannoni et al. (1960), Proença et al. (1935), Migliano et al. (1966), Neves et al. (1983), Marques et al. (1999), Paes-de-Almeida et al. (1999), Costa et al. (1988), Torres et al. (2001) e Silveira et al. (2005). Colpo et al. (2005) sugerem a hipótese de o próprio tropismo do parasito levá-lo ao rim direito, enquanto autores anteriores a estes, afirmam que as larvas chegam ao fígado pela corrente sanguínea e passam para o rim devido a 
proximidade destes onde se desenvolvem até o estágio adulto. Entretanto, a preferência pelo rim direito ainda não está totalmente esclarecida. Dacorso Filho et al. (1954), Migliano (1953), Neves (2005) e ainda Leite et al. (2005), Colpo et al. (2005) e Kommers et al. (1999) relatam hipertrofia compensatória do rim contra lateral, indo contra o achado do presente caso, onde o tal órgão não apresentou qualquer tipo de alteração. Monteiro et al. (2002) também sugeriram ausência de alterações em rim esquerdo.

Pela primeira vez, $D$. renale foi detectado na Baixada Santista de São Paulo, alertando para a importância do diagnóstico desta zoonose na região. Por ser uma animal proveniente da cidade de Pedro de Toledo, Sp, região banhada pelos rios Itariri, Espraiado, São Lourenço e Lourencinho, clima propício à ocorrência do parasita associado ao vasto acesso do hospedeiro definitivo, podem ter contribuído para a infecção no cão, uma vez que o parasita pode sobreviver até 5 anos dentro do rim do hospedeiro (Vibe, 1985). Já a cidade de Santos, possui manguezais, berço de várias espécies aquáticas e, apesar de nenhum registro da ocorrência de $\mathrm{D}$. renale, é uma hipótese que não pode ser descartada.

\section{Referências bibliográficas}

Barriga, O. (2002). Las enfermedades parasitarias de los animales domésticos em la América Latina. Santiago, Chile: Editorial Germinal, Santiago.

Beaver, P. C. \& Theis, J. H. (1979). Dioctophymatid larval nematode in a subcutaneous nodule from man in California. The American Journal of Tropical Medicine and Hygiene, 28(2), 206-212.

Colpo, C. B., Monteiro, S. G., Stainki, D. R., Colpo, E. T. B. \& Henriques, G. B. (2005). Natural infection by Trypanosoma evansi in dogs. Ciência Rural, 35(3), 717-719.

Costa, H.M.A.; Lima, W.S. 1988. Dioctophyme renale (Goeze, 1782): ocorrência em Minas Gerais. Arquivo Brasileiro de Medicina Veterinária e Zootecnia, 40, 43-245.

Cottar, B.H., Dittrich, G., Ferreira, A.A., Carvalho, A.C.P., Albernaz, V.G.P., Luz, M.T., Tasqueti, U.I. (2012). Achados ultrassonográficos de cães parasitados por Dioctophyma renale - Estudo retrospectivo. Veterinária e Zootecnia, 19(1), 8-11.

Dacorso Filho, P., Langenegger, J. \& Döbereiner, J. (1954). Sobre a infestação e lesões anatomo patológicas produzidas por Dioctophyme renale (Goeze, 1782) em cães. Veterinária, 8(2), 35-54.

Dyer, N. W. (1998). Dioctophyma renale in ranch mink. Journal of Veterinary Diagnostic Investigation, 10(1), 111-113.

Ferreira V.L., Medeiros F.P., July J.R. \& Raso T.F. (2010). Dioctophyma renale in a dog: clinical diagnosis and surgical treatment. Veterinary. Parasitology. 168(1/2):151-155.

Fossum, T. W. (2014). Cirurgia de pequenos animais (4 ed. Vol. 1). São Paulo: Elsevier Brasil.

Giovannoni, M. \& Molfi, A. O. (1960). Dioctophyme renale (Goeze, 1782) no Brasil. Anais da Faculdade de Medicina da Universidade do Paraná, 3, 99-144.

Ishizaki, M. N., Imbeloni, A. A., Muniz, J. A. P. C., Scalercio, S. R. R. A., Benigno, R. N. M., Pereira, W. L. A. \& Lacreta Junior, A. C. C. (2010). Dioctophyma renale (Goeze, 1782) in the abdominal cavity of a capuchin monkey (Cebus apella), Brazil. Veterinary Parasitology, 173(3-4), 340-343.

Kano, F. S., Shimada, M. T., Suzuki, S. N., Osaki, S. C., Menarim, B. C., Ruthes, F. R. V. \& Laidane Filho, M. A. (2003). Ocorrência da dioctofimose em dois cães no município de Guarapuava-PR. Semina: Ciências Agrárias, 24(1), 177-180.

Kommers, G. D., Ilha, M. R. S. \& Barros, C. S. L. (1999). Dioctofimose em cães\&58; 16 casos Dioctophymosis in dogs\&58; 16 cases. Ciência Rural, 29(3), 517-522.

Leite, L. C., Círio, S. M., Diniz, J. M. F., Luz, E., Navarro-Silva, M. A., Silva, A. W. C., . . Veronesi, E. M. (2005). Lesões anatomopatológicas presentes na infecção por Dioctophyma renale (Goeze, 1782) em cães domésticos (Canis familiaris, Linnaeus, 1758). Archives of Veterinary Science, 10(1), 95-101.

Marques, A.P., Raffi, L.L., Raffi, M.B., Lopes, D.C., Souza, R.B. \& Raposo, J.B. (1999). Dioctophyma renale em caninos no município de Pelotas-R.S - relato de caso, Escola de Veterinária da UFMG, 61.

Mascarenhas, C. S. \& Müller, G. (2015). Third-stage larvae of the enoplid nematode Dioctophyme renale (Goeze, 1782) in the freshwater turtle Trachemys dorbigni from southern Brazil. Journal of Helminthology, 89(5), 630-635.

Measures, L. N. \& Anderson, R. C. (1985). Centrarchid fish as paratenic hosts of the giant kidney worm, Dioctophyma renale (Goeze, 1782), in Ontario, Canada. Journal of Wildlife Diseases, 21(1), 11-19. 
Mech, D. L. \& Tracy, S. P. (2001). Prevalence of giant kidney worm (Dioctophyma renale) in wild mink (Mustela vison) in Minnesota. The American Midland Naturalist, 145(1), 206-209.

Migliano, M. F. (1953). Uremia em cães. Revista da Faculdade de Medicina Veterinária, Universidade de São Paulo, 5(1), 157-172.

Migliano, M.F. \& Matera, E. A. (1966). Dioctofimose em Canis familiaris, estudo clínico médico e cirúrgico de um caso. Revista de Medicina Veterinária, São Paulo, 2(2), 81-93.

Milanelo, L., Moreira, M. B., Fitorra, L. S., Petri, B. S. S., Alves, M. \& Santos, A. d. C. (2009). Occurrence of parasitism by Dioctophyma renale in ring-tailed coatis (Nasua nasua) of the Tiete Ecological Park, São Paulo, Brazil. Pesquisa Veterinária Brasileira, 29(12), 959-962.

Miranda, M. A., Benigno, R. N., Galväo, G. R. \& Oliveira, S. A. L. (1992). Dioctophyme renale (Goeze, 1782): localizaçäo ectópica e alta intensidade parasitária em Canis familiaris do Pará-Brasil. Arquivo Brasileiro de Medecina Veterinária e Zootecnia, 44(2), 151-153.

Monteiro, S. G., Sallis, E. S. V. \& Stainki, D. R. (2002). Infecção natural por trinta e quatro helmintos da espécie Dioctophyma renale (Goeze, 1782) em um cão. Revista da FZVA, 9(1), 9.

Nakagawa, T. L. D. R., Bracarense, A. P. F. R. L., Reis, A. C. F., Yamamura, M. H. \& Headley, S. A. (2007). Giant kidney worm (Dioctophyma renale) infections in dogs from Northern Paraná, Brazil. Veterinary Parasitology, 145(3-4), 366-370.

Neves, D. P. (2005). Parasitologia humana. São Paulo, Brasil: Editora Atheneu.

Neves, D. S., Morais, A.N., Nogueira, R.H.G. \& Chquiloff, M.A.G. (1983). Ocorrência de Dioctophyme renale (Goeze, 1782) em cães da região de Lages, Estado de Santa Catarina. Arquivo Brasileiro de Medicina Veterinária e Zootecnia, Belo Horizonte, 35(5), 665-773.

Paes-de-Almeida, E.C. \& Thomé, S.M.G. (1999). Ocorrência da dioctofimose (Dioctophyma renale) canina no município de Silva Jardim - RJ. Escola de Veterinária da UFMG, 65.

Pereira, B. J., Girardelli, G. L., Trivilin, L. O., Lima, V. R., Nunes, L. C. \& Martins, I. V. F. (2006). Ocorrência de dioctofimose em cães do município de Cachoeiro do Itapemirim, Espírito Santo, Brasil, no período de maio a dezembro de 2004. Revista Brasileira de Parasitologia Veterinária, 15(3), 123-125.

Proença, M.C. (1935). Sobre dois casos de Dioctophyme renale em cão do Rio de Janeiro. Boletim Veterinário do Exército., Rio de Janeiro, 2(3), 50-51.

Santos, V. A. \& Beisiegel, B. M. (2006). A dieta de Nasua nasua (Linnaeus, 1766) no Parque Ecológico do Tietê, SP1. Revista Brasileira de Zoociências, 8(2), 199-203.

Silveira, C.S., Diefenbach, A., Mistieri, M.L., Machado, I.R.L. \& Anjos, B.L. (2005). Dioctophyma renale em 28 cães: aspectos clinicopatológicos e ultrassonográficos. 2005. Pesquisa Veteterinária Brasileira. 35(11), 899-905.

Torres, R., Alves, A., Borges, A.G., Rahal, S.C. \& Sakate, M. (2001). Dioctofimíase canina. Ciência Animal. 11(1), p. 197.

Verocai, G. G., Measures, L. N., Azevedo, F. D., Correia, T. R., Fernandes, J. I. \& Scott, F. B. (2009). Dioctophyme renale (Goeze, 1782) in the abdominal cavity of a domestic cat from Brazil. Veterinary Parasitology, 161(3-4), 342-344.

Vibe, P. P. (1985). Dioctophyma infection in Humans. Medical Parazitology, 1, 83-84.

Zabott, M. V., Pinto, S. B., Viott, A. M., Tostes, R. A., Bittencourt, L. H. F. B., Konell, A. L. \& Gruchouskei, L. (2012). Occurrence of Dioctophyma renale in Galictis cuja. Pesquisa Veterinária Brasileira, 32(8), 786-788.

Zardo, K.M., Santos, D.R., Babicsak, V.R., Belotta, A.F., Oliveira, H.S., Estanislau, C.A., Mamprim, M.J. \& Brandão, C.V.S. (2012). Aspecto ultrassonográfico da dioctofimose renal canina. Veterinária e Zootecnia.19(1):57-60.

Recebido: 18 novembro, 2018.

Aprovado: 10 dezembro, 2018 .

Publicado: 25 janeiro, 2019.

Licenciamento: Este artigo é publicado na modalidade Acesso Aberto sob a licença Creative Commons Atribuição 4.0 (CCBY 4.0), a qual permite uso irrestrito, distribuição, reprodução em qualquer meio, desde que o autor e a fonte sejam devidamente creditados 